\title{
Towards Realistic Mobility Models for Vehicular Ad-hoc Networks
}

\author{
Rainer Baumann*, Simon Heimlicher*, Martin May* \\ ${ }^{*}$ Computer Engineering and Networks Laboratory \\ ETH Zurich, Switzerland
}

\begin{abstract}
In performance studies of vehicular ad hoc networks, the underlying mobility model plays a major role. In this paper, we investigate the influence of three mobility models on the performance of ad hoc network routing protocols (AODV and GPSR). As a benchmark, we use the popular random waypoint mobility model. Our second model is based on a vehicular traffic simulator that we proposed in earlier work. Finally, as third model, we propose a novel mobility model based on vectorized street maps and speed limit information extracted from a geographic information system. With the two considered routing protocols, the random waypoint mobility model tends to lead to substantially higher performance than with our own, presumably more realistic mobility models.
\end{abstract}

\section{INTRODUCTION}

The evaluation of protocols and algorithms for vehicular ad hoc networks is based mostly on theory or simulation experiments as large-scale testbeds would be too expensive to be practical. However, high predictability can be expected from simulation studies only if the mobility model resembles reality closely.

In this paper, we investigate the impact of the mobility model choice on the routing protocol performance. We propose two mobility models and use the random waypoint mobility model as a benchmark. The first mobility model we present uses vectorized street information, including speed limit data, from the Swiss Geographic Information System (GIS) [1]. The second model is based on traces from a microscopic, multiagent traffic simulator that we have presented in our previous work [2].

Both mobility models have advantages and limitations. The GIS-based model requires only little computing power but the scenarios it produces are not as close to reality as those of the trace-based model. In contrast, the trace-based model imitates reality very closely, but requires a huge amount of computing power for the generation of traces.

In order to determine the influence of the mobility model on the performance of routing, we consider two popular routing protocols AODV [3] and GPSR [4]. These routing protocols represent two major classes of ad hoc routing protocols: AODV represents reactive, non-geographic routing, whereas GPSR represents geographic routing with greedy forwarding. Both AODV and GPSR are well documented, have been tested in many research studies and are known as good performers in their classes of routing protocols.

Our results indicate a significant drop of the packet delivery ratio with our presumably more realistic models compared to the delivery ratio achieved with the random waypoint model.

\section{RELATED WORK}

Analysis of related literature reveals that the results of performance studies of ad hoc networks depend heavily on the chosen mobility model [5]-[8]. We distinguish two categories of mobility models. First, there are simple mobility models like random waypoint, random directions, Manhattan, and other models where the nodes change their speed and direction randomly. Second, there are more complex models where the movement and behavior of nodes are modeled closer to reality [2], [8]-[12]. Results obtained with models from different category often differ significantly. This discrepancy raises the question whether the simple models are consistent enough with reality.

One realistic modeling approach would be to use mobility traces. However, no traces are available for pedestrians or vehicles moving around in cities. There are traces for buses of public transport systems [13], but these do not necessarily match the mobility of pedestrians or cars. As a result, the mobility of vehicles is often approximated by random mobility models configured with higher maximum node speed [14]. Sawant et al. [15] describe vehicular movement on a highway by simulating a set of nodes moving at a constant speed along the longer side of a rectangular area of $1000 \mathrm{~m} \times 100 \mathrm{~m}$. A slightly more advanced model is used in [16], where the decisions of the car drivers are included in the mobility model.

Saha and Johnson proposed in [9] to use street maps form the TIGER database to generate quite realistic mobility patterns. However, their model neither follows the exact geometry of the streets nor does it incorporate speed limits. In Section III we present a new mobility model that is based on exact vectorized street maps of major Swiss cities with complete speed limit information.

Lochert et al. [10] use the traffic flow simulator Videlio developed by Daimler Chrysler AG to create a movement pattern for a small part $(6.25 \mathrm{~km} \times 3.45 \mathrm{~km})$ of the city of Berlin. No information is given on how the density/distribution 
of vehicles were chosen. The authors consider only one area of the city and 10 data sources that are active for only 5 seconds. Choffnes et al. proposed STRAW [8], a simple microscopic traffic simulator based on the TIGER Street maps. In Section IV we describe a source of realistic vehicular mobility traces that we proposed in [2]. These traces are obtained from a multi-agent microscopic traffic simulator that was developed by K. Nagel (at ETH Zurich, now with the Technical University in Berlin, Germany). In contrast to STRAW, this simulator is capable of simulating public and private vehicular traffic over real regional road maps of Switzerland with a high level of realism [11], [12]. The multi-agent core enables accurate reproduction of the behavior of the individuals, such as the choice of means of transportation. Moreover, the start time of a trip and its destination is considered for every single person in the simulation.

\section{Mobility Model BASEd ON GIS DATA}

With this mobility model, we aim to mimic the movement of pedestrians and vehicles. To this end, we constrain the movement of the nodes to streets. We extract street maps of real Swiss cities from the Swiss Geographic Information System (GIS) [1]. This database includes vectorized building and street maps including speed limit information and many other data. As an example, the vectorized maps of the city centers of four large Swiss cities are displayed in Fig. 11. A major advantage of this data source is its high level of detail. The precision of the street geometry is 1 meter, thus even small turns and the number of lanes are mapped. An other advantage is the availability of an elevation profile as well as additional information about the maximum velocities.

In our GIS-based mobility model, the actual node movement is generated according to the steady-state random trip mobility model [17] on the vectorized street maps as follows. A node chooses a random destination in the city and moves to this position using the fastest available path, i.e., the shortest path in terms of time. In order to determine this path, we calculate a weighted shortest path [18] whose edges are weighted according to the time required to pass through a street segment at the maximal allowed speed. The model captures the behavior of pedestrians as well as cars since both move along the streets of a city. We generate two scenarios: (i) a scenario with mobile nodes moving at pedestrian speeds (i.e., node speeds that are uniformly distributed between $1 \mathrm{~m} / \mathrm{s}$ and $4 \mathrm{~m} / \mathrm{s}$ ) and (ii) a scenario with nodes moving at car speeds according to the maximal allowed speed on that street segment. In order to account for cars that do not move at the maximal speed, the speeds are uniformly distributed between $75 \%$ and $100 \%$ of the speed limit, corresponding to speeds of approximately $10 \mathrm{~m} / \mathrm{s}-20 \mathrm{~m} / \mathrm{s}$.

\section{Mobility Model using Multi-Agent Microscopic TrafFic Simulator}

Since real vehicular traces are not available, a traffic simulator can be used to generate the movement of vehicles. However, driver behavior on a road is very complex since it depends on the environment. Drivers react to changing road conditions such as for instance congestion, which in turn depend on the drivers' plans and behavior. Furthermore, weather and other environmental factors also influence the behavior of the individuals. Thus, the choice of the traffic simulator in the end determines the relevance and viability of the obtained results.

Vehicular traffic simulators can in general be classified into microscopic and macroscopic simulators. A macroscopic simulator considers such system parameters as traffic density (number of vehicles per km per lane) or traffic flow (number of vehicles per hour crossing some point, usually an intersection) to compute road capacity and the distribution of the traffic in the road network. From the macroscopic perspective, vehicular traffic is viewed as a fluid compressible medium, and therefore is modeled based on an equation deduced from the NavierStokes equations [19]. In contrast, microscopic simulators determine the movement of each vehicle that participates in the road traffic. Thus, a microscopic traffic simulator is potentially a better choice for our research.

The Multi-agent Microscopic Traffic Simulator (MMTS) [11], [12] developed at ETH Zurich is capable of simulating public and private traffic over real regional road maps of Switzerland with a high level of realism. MMTS models the behavior of people living in the area, reproducing their movement (using vehicles) within a period of 24 hours. The decision of each individual depends on the area it lives in. The individuals in the simulation are distributed over the cities and villages according to statistical data gathered by a census. Within the 24 hours of simulation, all individuals choose a time to travel and the mean of transportation according to their needs and environment. For instance, one individual might board their car and go to work in the early morning, another one that gets up later goes shopping using public transportation, etc. Travel plans are made based on road congestion; congestion in turn depends on the travel plans. To resolve this situation, a standard relaxation method is used [20], [21].

The street network that is used in MMTS was originally developed for the Swiss regional planning authority (Federal Office of Spatial Development). The major attributes of each road segment are type, length, speed, and capacity. The street network is simulated on a Beowulf [22] Pentium cluster of over 30 CPUs. With the help of MMTS, the consequences of construction sites, road modifications, new roads, etc. can be simulated and potential economical influence (e.g., travel time and price changes for public and private transport) can be estimated. 

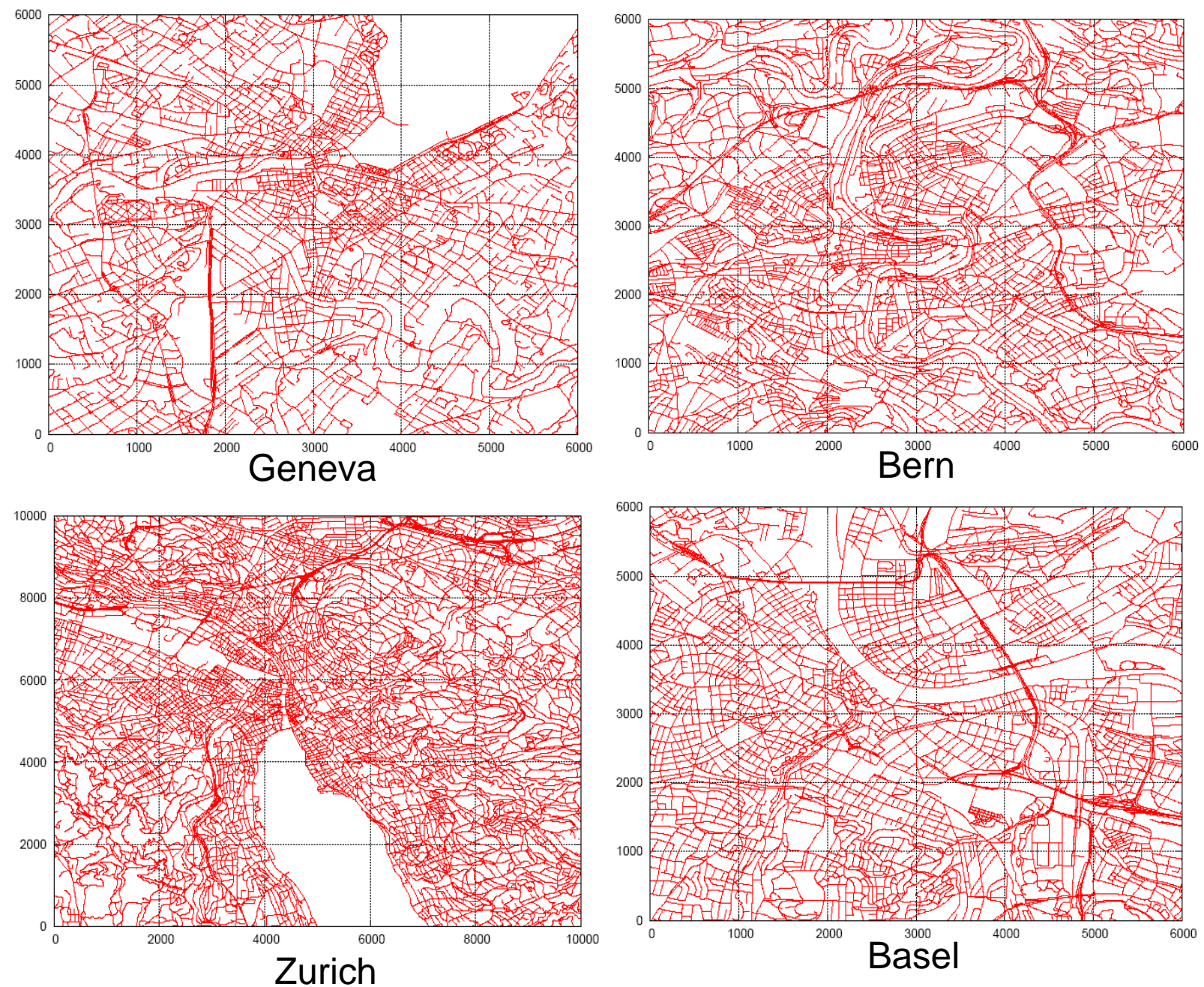

Fig. 1. Vectorized street map of four Swiss cities.

For generating vehicular mobility traces, we use a 24hour, detailed car traffic trace file generated by MMTS. The file contains detailed simulation data of a certain geographic region in the canton of Zurich. This region includes the part where the main country highways connect to the city of Zurich, the largest city in Switzerland. Around 260'000 vehicles are involved in the simulation with more than $25^{\prime} 000^{\prime} 000$ recorded direction/speed changes in an area of around $250 \mathrm{~km}$ $\times 260 \mathrm{~km}$ (see Figure 2).

The car traffic simulator file describes in XML format the step by step movement of each vehicle within the 24-hour time period. To use that data we convert the XML input from the car traffic simulator into simulator movement pattern. However, the file resulting from car traffic simulator movement pattern contains too many nodes (vehicles) to be processed by any available network simulator. Thus to allow simulation on a network simulator, we select smaller subregions that are suitable for simulations as shown in Figure 3. The selected region contains the 24 hour movement pattern of all the vehicles that travel inside or through a region. As a next step we capture different levels of activity of vehicles in the region (the number of events per time slot) and select three time periods that correspond to high density rush hour (more than 50 vehicles per $\mathrm{km}$ of road), medium density (30-40 vehicles/ $\mathrm{km}$ ), and low vehicle density (less than 15 vehicles $/ \mathrm{km}$ ). Each period has a length of 0.5 hours. Finally, from each of these periods, we create 10 scenario files of 300 seconds, each shifted by 150 seconds from the previous one.

In Figure 4 a snapshot of mobile nodes (vehicles) moving about the center of Zurich-city is superimposed above a map of the region.

\section{Simulation Study}

We now discuss the influence of the chosen mobility model on the performance of two popular routing protocols: AODV and GPSR. For this comparison, we use the two proposed mobility models as well as the random waypoint mobility model. Before presenting the results, we describe the simulation and mobility model set-up. 


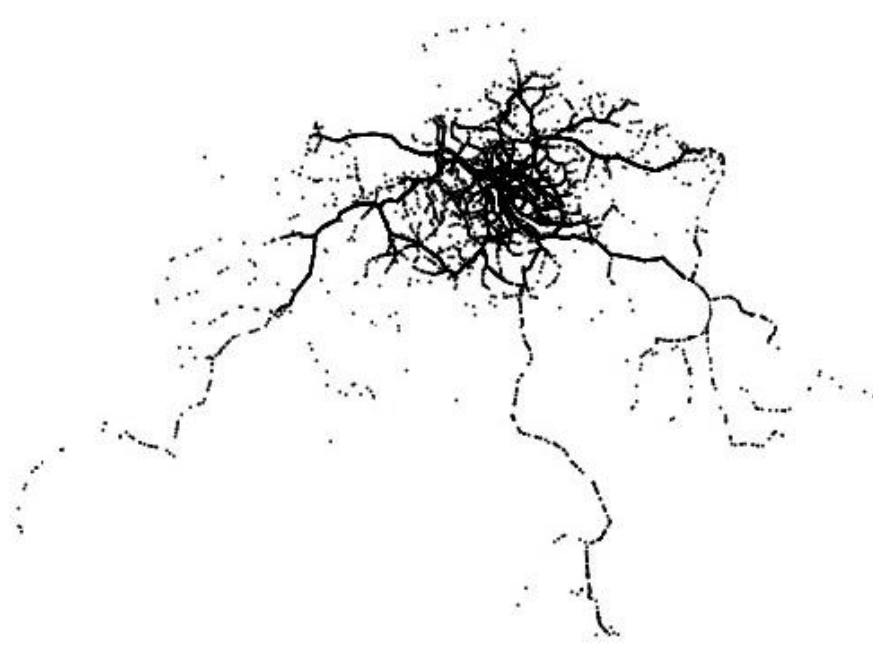

Fig. 2. Road network and car traffic in north-eastern Switzerland.

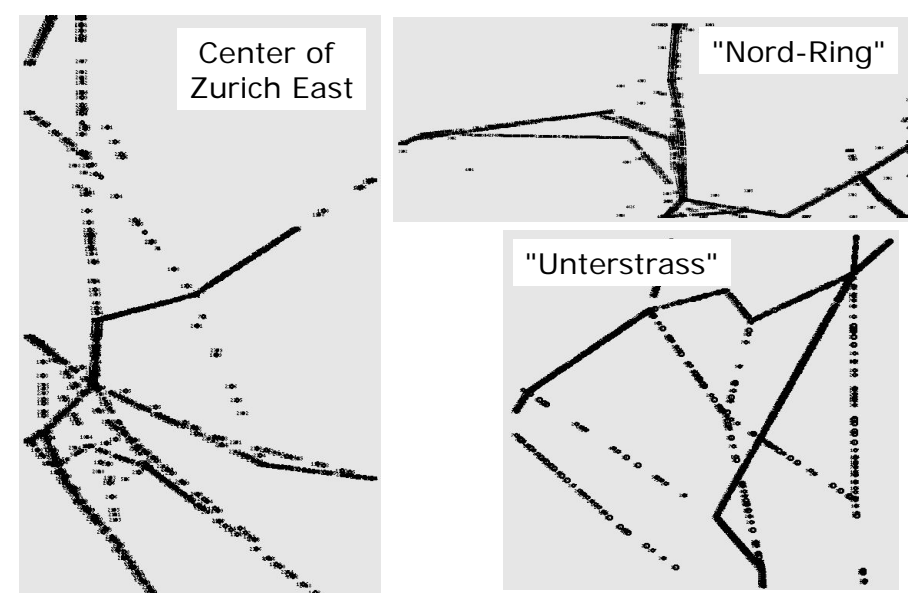

Fig. 3. Visualization examples of different inner city regions of Zurich.

\section{A. Simulation Set-up}

The literature shows that the results of performance studies of ad hoc network depend heavily on the chosen mobility model [5]-[7]. To allow our study to be compared with prior work, we investigate AODV and GPSR ${ }^{1}$ based on the random waypoint mobility model [24] and compare the results with our mobility models using release 2.31 of the ns-2 [25] network simulator. For the simulations, we use the default settings for the GPSR and AODV routing protocols.

To gain more realistic signal propagation than with the deterministic free space or two-ray ground reflection model, we use the shadowing model for radio propagation [26]. According to real world experiments with inter-vehicle communication [27], [28], we choose $\beta=3.5$ and $\sigma_{d B}=6$. All nodes are equipped with a $802.11 \mathrm{~b}$ radio with a bandwidth of $11 \mathrm{Mbps}$ and a nominal range of 250 meters. As MAC layer

\footnotetext{
${ }^{1}$ The code for GPSR is taken from [23].
}

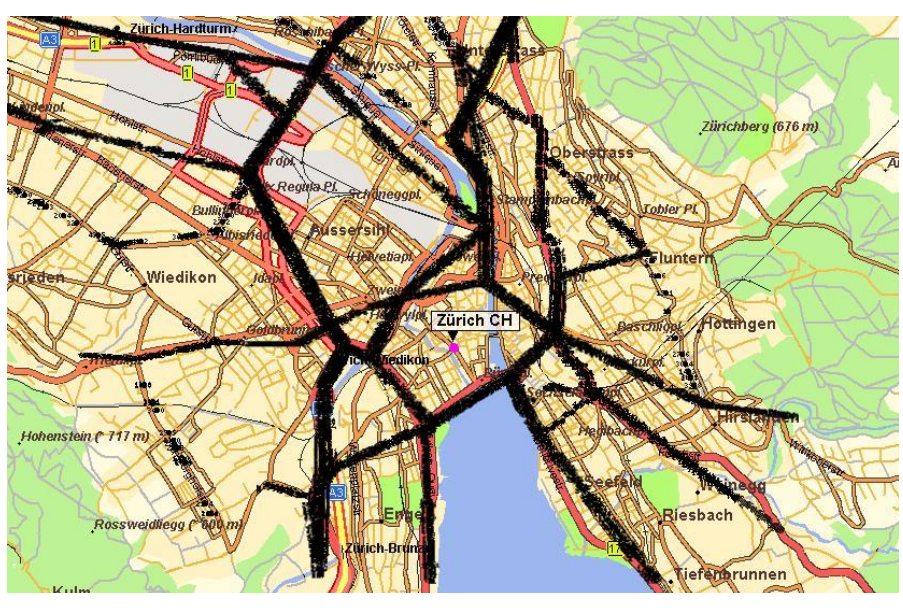

Fig. 4. Superposition of mobility pattern on a map of the center of Zurich.

protocol, we use the 802.11 DFWMAC-DCF w/RTS/CTS [29].

Traffic sources transmit data at a fixed data rate. We are not interested in the maximum achievable throughput in VANET; we rather want to investigate the influence of the different mobility models on the performance, i.e. on the packet delivery ratio. Thus we consider randomly 25 source-destination pairs with a sending rate of $100 \mathrm{kbit} / \mathrm{s}$. The sources start generating data packets within the first $50 \mathrm{~s}$ of the simulation time and stop generating data packets $50 \mathrm{~s}$ before the simulation ends to avoid transient effects on our results.

\section{B. Set-up of Mobility Models}

For the random waypoint model we simulate an area of $5 \mathrm{~km} \times 5 \mathrm{~km}$ populated by 1100 nodes that move about the area. The node speeds vary between $15 \mathrm{~km} / \mathrm{h}$ and $55 \mathrm{~km} / \mathrm{h}$. The pause time equals $0 \mathrm{~s}$ (constant motion), $200 \mathrm{~s}$ and 300 s. For the mobility model using GIS-data, we select the same area size, the same number of nodes using the scenario with nodes moving at car speed and the same pausing times as for random waypoint. Also for the vehicular traces we select the same area size representing the city region. We identify the $300 \mathrm{~s}$ time interval when the selected area is populated by at least 1000 nodes and at most by 1200 nodes. Pause time is modeled like follows: for $300 \mathrm{~s}$ pause time (static picture) we cancel all node mobility; for $200 \mathrm{~s}$ pause time we keep nodes static for $200 \mathrm{~s}$ and then allow movement; for $0 \mathrm{~s}$ pause time no restrictions are applied.

\section{Results}

Figures 5 and 6 show the packet delivery ratio of AODV and GPSR with the random waypoint model and with our more realistic models. The performance of both protocols shows noticeable dependence on the chosen mobility model. With the random waypoint model, GPSR outperforms AODV; with the vehicular traces the situation changes to the opposite 


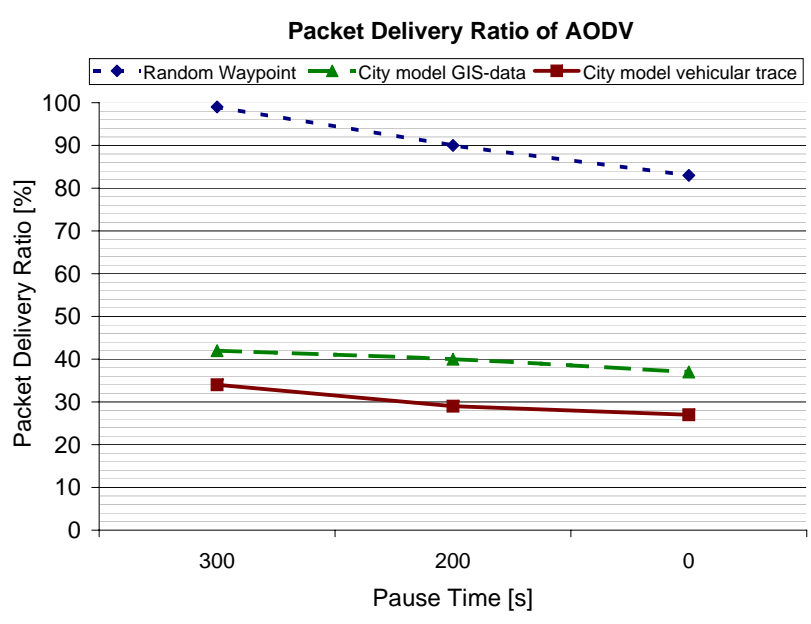

Fig. 5. Packet Delivery Ratio of AODV

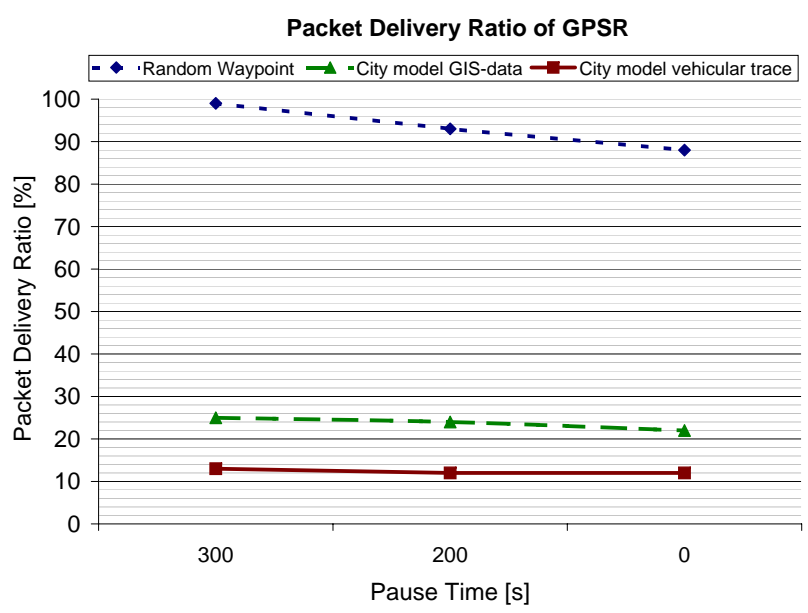

Fig. 6. Packet Delivery Ratio of GPSR

(see tables I and II). Using the proposed mobility models, the packet delivery ratio of both protocols turns out to be much lower than in the corresponding random waypoint model scenarios.

Comparing the two proposed mobility models shows that the performance using the GIS-data based model is close to the performance of the model based on the traffic simulator traces. Since the GIS-data based model requires much fewer computing resources for generating mobility patterns than the trace-based model and still provides quite similar results, it provides a viable alternative.

Our analysis of the degraded packet delivery ratio compared to random waypoint has revealed the following main causes.

1) We found that with both realistic models, the average path length (in number of hops) is around 1.3 to 2 times longer than with the random waypoint model. This increased path length is due to the restriction of the node movements to the roads, which leads to routes with detours. These detours increase the average path length, which incurs a higher packet loss ratio.

\begin{tabular}{|c|c|c|c|}
\hline & $\begin{array}{c}\text { Random } \\
\text { waypoint }\end{array}$ & $\begin{array}{c}\text { City model } \\
\text { GIS-data }\end{array}$ & $\begin{array}{c}\text { City model } \\
\text { vehicular trace }\end{array}$ \\
\hline Pause time & AODV & AODV & AODV \\
\hline $300 \mathrm{~s}$ & 99 & 42 & 34 \\
\hline $200 \mathrm{~s}$ & 90 & 40 & 29 \\
\hline $0 \mathrm{~s}$ & 83 & 37 & 27 \\
\hline
\end{tabular}

TABLE I

PACKET DELIVERY RATIO OF AODV.

\begin{tabular}{|c|c|c|c|}
\hline & $\begin{array}{c}\text { Random } \\
\text { waypoint }\end{array}$ & $\begin{array}{c}\text { City model } \\
\text { GIS-data }\end{array}$ & $\begin{array}{c}\text { City model } \\
\text { vehicular trace }\end{array}$ \\
\hline Pause time & GPSR & GPSR & GPSR \\
\hline $300 \mathrm{~s}$ & 99 & 25 & 13 \\
\hline $200 \mathrm{~s}$ & 93 & 24 & 12 \\
\hline $0 \mathrm{~s}$ & 88 & 22 & 12 \\
\hline
\end{tabular}

TABLE II

PACKET DELIVERY RATIO OF GPSR.

2) With the random waypoint mobility model, nodes move along a straight line from a starting point to a destination at a constant speed. In contrast, the two novel models force nodes to change their direction and speed more frequently. This leads to an increased link breakage ratio, mainly for nodes close to the maximal communication range, and also increases the packet loss ratio.

\section{CONCLUSion AND Future Work}

In this paper, we have proposed two novel mobility models that model reality more closely than simple models such as random waypoint. Our first model is based on street maps with speed limit information from the Swiss geographic information system. The second model is based on realistic traces from a microscopic multi-agent traffic simulator. The latter model provides potentially more realistic mobility patterns. However, it requires much more computing resources than the first model.

Our evaluation has validated the finding that performance results of vehicular ad hoc networks depend highly on the underlying mobility model. The simple random waypoint mobility model tends to considerably overestimate the performance of the routing protocols that we used for our evaluation (AODV and GPSR).

We conclude from our results that care should be taken if simple mobility models are used for the evaluation of vehicular ad hoc networks as the results obtained with these models might not be as close to reality as expected. One striking difference between the simple random waypoint model and our more complex mobility models is that our models lead to considerably longer paths and this in turns contributes to the higher packet loss ratio seen with our models.

As further work we mainly plan to investigate different routing protocols. Furthermore, we are considering to further investigate the differences between the mobility models we propose in this paper. In particular, we are interested to determine if the difference in the results of our models can be attributed to a particular property of the GIS-based model. 


\section{REFERENCES}

[1] Federal Office of Topography, "Swiss Geographic Information System." [Online]. Available: www.swisstopo.ch

[2] V. Naumov, R. Baumann, and T. Gross, "An evaluation of inter-vehicle ad hoc networks based on realistic vehicular traces," in MobiHoc '06: Proceedings of the seventh ACM international symposium on Mobile ad hoc networking and computing, 2006, pp. 108-119.

[3] C. E. Perkins, E. M. Belding-Royer, and S. R. Das, "Ad Hoc OnDemand Distance Vector (AODV) Routing," IETF Internet Draft, draftietf-manet-aodv-12.txt, November 2002.

[4] B. Karp and H. Kung, "GPSR: Greedy Perimeter Stateless Routing for Wireless Networks," in Proceedings of the 6th Annual ACM/IEEE International Conference on Mobile Computing and Networking (MobiCom), Boston, US, August 2000.

[5] J. Yoon, M. Liu, and B. Noble, "Random waypoint considered harmful." in INFOCOM, 2003.

[6] C. Bettstetter, G. Resta, and P. Santi, "The Node Distribution of the Random Waypoint Mobility Model for Wireless Ad Hoc Networks," IEEE Transactions on Mobile Computing, vol. 2, no. 3, pp. 257-269, July-September 2003.

[7] T. Camp, J. Boleng, and V. Davies, "A survey of mobility models for ad hoc network research," Wireless Communications \& Mobile Computing (WCMC): Special issue on Mobile Ad Hoc Networking: Research, Trends and Applications, vol. 2, no. 5, pp. 483-502, 2002. [Online]. Available: citeseer.ist.psu.edu/camp02survey.html

[8] D. R. Choffnes and F. ann E. Bustamante, "An integrated mobility and traffic model for vehicular wireless networks," in VANET '05: Proceedings of the 2nd ACM international workshop on Vehicular ad hoc networks. New York, NY, USA: ACM Press, 2005, pp. 69-78.

[9] A. Saha and D. Johnson, "Modeling mobility for vehicular ad hoc networks," in In poster at ACM VANET, 2004

[10] C. Lochert, H. Hartenstein, J. Tian, H. Füßler, D. Herrmann, and M. Mauve, "A Routing Strategy for Vehicular Ad Hoc Networks in City Environments," in Proc. of IEEE Intelligent Vehicles Symposium (IV2003), Columbus, OH, 06 2003, pp. 156-161.

[11] B. Raney, N. Cetin, A. Vollmy, M. Vrtic, K. Axhausen, and K. Nagel, "An agentbased microsimulation model of swiss travel: First results," 2003. [Online]. Available: citeseer.ist.psu.edu/raney03agentbased.html

[12] B. Raney, A. Voellmy, N. Cetin, M. Vrtic, and K. Nagel, "Towards a microscopic traffic simulation of all of switzerland," in International Conference on Computational Science (1), 2002, pp. 371-380. [Online]. Available: citeseer.ist.psu.edu/568749.html

[13] J. Jetcheva, Y.-C. Hu, S. PalChaudhuri, A. K. Saha, and D. B. Johnson, "Design and evaluation of a metropolitan area multitier wireless ad hoc network architecture," in Proceedings of the 5th IEEE Workshop on Mobile Computing Systems \& Applications (WMCSA), Monterey, CA, October 2003.

[14] R. Morris, J. Jannotti, F. Kaashoek, J. Li, and D. Decouto, "Carnet: a scalable ad hoc wireless network system," in EW 9: Proceedings of the 9th workshop on ACM SIGOPS European workshop, 2000, pp. 61-65.

[15] H. Sawant, J. Tan, and Q. Yang, "Study of an inter-vehicle communication protocol for vehicle-infrastructure integration (vii)," in In Transportation Research Board 84th Annual Meeting, 2005.

[16] Z. D. Chen, H. Kung, and D. Vlah, "Ad hoc relay wireless networks over moving vehicles on highways," in MobiHoc '01: Proceedings of the 2nd ACM international symposium on Mobile ad hoc networking \& computing. New York, NY, USA: ACM Press, 2001, pp. 247-250.

[17] J.-Y. Le Boudec and M. Vojnovic, "Perfect Simulation and Stationarity of a Class of Mobility Models," in IEEE Infocom, 2005.

[18] R. K. Ahuja, T. L. Magnanti, and J. B. Orlin, Network Flows: Theory, Algorithms, and Applications. Prentice Hall, 1993, aHU r 93:1 P-Ex.

[19] L. Landau and E. M. Lifshitz, Fluid Mechanics. Pergamon Press, 1987.

[20] J. H. Mathews, Numerical methods for mathematics, science, and engineering, 2nd ed., 1992, rev. ed. of: Numerical methods for computer science, engineering, and mathematics. 1987.

[21] W. H. Press, "Modeling of data," in Numerical Recipes in C. The Art of Scientific Computing. Cambridge University Press, 1992, pp. 657-706.

[22] D. Becker and P. Merkey, "The Beowulf Project," 2002. [Online]. Available: http://www.beowulf.org/
[23] B. Karp and H. T. Kung, "Gpsr: greedy perimeter stateless routing for wireless networks," in MobiCom '00: Proceedings of the 6th annual international conference on Mobile computing and networking. New York, NY, USA: ACM Press, 2000, pp. 243-254.

[24] J. Broch, D. A. Maltz, D. B. Johnson, Y.-C. Hu, and J. Jetcheva, "A performance comparison of multi-hop wireless ad hoc network routing protocols," in Mobile Computing and Networking, 1998, pp. 85-97. [Online]. Available: citeseer.ist.psu.edu/broch98performance.html

[25] "The Network Simulator - ns-2," http://www.isi.edu/nsnam/ns/, 2002.

[26] T. S. Rappaport, Wireless Communications: Principles and Practice. Piscataway, NJ, USA: IEEE Press, 1996.

[27] N. Eude, B. Ducourthial, , and M. Shawky, "Enhancing ns-2 simulator for high mobility ad hoc networks in car-to-car communication context," in MWCN'05, 2005.

[28] J. Singh, N. Bambos, B. Srinivasan, and D. Clawin, "Wireless lan performance under varied stress conditions in vehicular traffic scenarios," 2002. [Online]. Available: citeseer.ist.psu.edu/ singh02wireless.html

[29] IEEE Std 802.11 , 1997-2007, Information Technology- telecommunications And Information exchange Between Systems-Local And Metropolitan Area Networks-specific Requirements-part 11: Wireless Lan Medium Access Control (MAC) And Physical Layer (PHY) Specifications. New York, NY: Institute of Electrical and Electronic Engineers, Inc., 19992007. 